\title{
RF CMOS inductor shielded by a high-impedance surface
}

\author{
Eiichi Sano, ${ }^{\text {a) }}$ Koji Inafune, and Masamichi Akazawa \\ Research Center for Integrated Quantum Electronics, Hokkaido University \\ North 13, West 8, Kita-ku, Sapporo 060-8628, Japan \\ a)esano@rciqe.hokudai.ac.jp
}

\begin{abstract}
We propose a high-performance inductor based on the concept of a high-impedance surface (or artificial magnetic conductor). The inductor is shielded from the silicon substrate by a high-impedance surface consisting of a lumped capacitor and inductor. Theoretical calculations comparing the proposed inductor with a conventional inductor shielded by an ordinary metal surface show that the resonant frequency and quality factor $(Q)$ in the millimeter-wave region are higher for the proposed inductor.
\end{abstract}

Keywords: inductor, RF CMOS, high-impedance surface, artificial magnetic conductor, quality factor

Classification: Microwave and millimeter wave devices, circuits, and systems

\section{References}

[1] C. Patrick Yue and S. Simon Wong, "On-chip spiral inductors with patterned ground shields for Si-based RF IC's," IEEE J. Solid-State Circuits, vol. 33, no. 5, pp. 743-752, 1998.

[2] D. Sievenpiper, L. Zhang, R. Broas, N. G. Alexopolous, and E. Yablonovitch, "High-impedance electromagnetic surfaces with a forbidden frequency band," IEEE Trans. Microwave Theory Tech., vol. 47, no. 11, pp. 2059-2074, 1999.

[3] F.-R. Yang, K.-P. Ma, Y. Qian, and T. Itoh, "A novel TEM waveguide using uniplanar compact photonic-bandgap (UC-PBG) structure," IEEE Trans. Microwave Theory Tech., vol. 47, no. 11, pp. 2092-2098, 1999.

[4] Y. Zhang, J. von Hagen, M. Younis, C. Fischer, and W. Wiesbeck, "Planar artificial magnetic conductors and patch antennas," IEEE Trans. Antennas Propagat., vol. 51, no. 10, pp. 2704-2712, 2003.

\section{Introduction}

CMOS-compatible inductors with a high quality factor $(Q)$, which should be useful in high-performance microwave and millimeter wave integrated circuits, cannot be made without reducing their parasitic resistance and substrate loss. This is usually done by using a multi-layer structure in which ground plates shield the inductor from the silicon substrate. Eddy currents on the ground plates degrade $Q$, however, even when patterned ground shields 
are used to block them [1]. On the other hand, high-impedance surfaces [2], [3] and artificial magnetic conductors [4] have been used successfully in antenna reflectors. Arrays of patch elements (distributed circuit elements) have been used there, but the large size of distributed circuit elements makes them hard to use at frequencies below a few tens of gigahertz.

This letter therefore describes a new inductor that is shielded from the silicon substrate by a high-impedance surface consisting of a lumped capacitor and inductor. It also reports the results of theoretical calculations comparing the characteristics for this inductor and conventional inductors.

\section{An inductor with high-impedance surface}

Structures and equivalent circuits for the conventional and proposed inductors are shown in Fig. 1. The substrate loss described in Fig. 1 (a) by $G_{\text {sub }}$ and $C_{\text {sub }}$ is often eliminated by a shield metal placed on the field oxide as shown in Fig. 1 (b). The quality factor $Q$ for the equivalent circuit shown in Fig. 1 (b) is given by

$$
Q=\frac{\omega}{r_{i}}\left(L_{i}-r_{i}^{2} C_{i}-\omega^{2} L_{i}^{2} C_{i}\right)
$$

where $\omega$ is the angular frequency. The resonant frequency $\omega_{0}$ at which $Q=0$ is given by

$$
\omega_{0}=\sqrt{\frac{L_{i}-r_{i}^{2} C_{i}}{L_{i}^{2} C_{i}}} .
$$

$Q$ increases with increasing $\omega$ below $\omega_{0} / \sqrt{3}$ and then decreases because of the parasitic capacitance $C_{i}$. When the shield metal is replaced by a highimpedance surface (HIS), the equivalent circuit is the one shown in Fig. 1 (c). At $\omega_{h}=1 / \sqrt{L_{h} C_{h}}$, the impedance of the HIS is infinite. The parasitic capacitance $C_{i}$ has no influence on the inductor $Q$ at $\omega_{h}$, where $Q$ is given by $\omega_{h} L_{i} / r_{i}$, but the $Q$ of an actual inductor is reduced by a parasitic resistance $r_{h}$ in series with $L_{h}$.

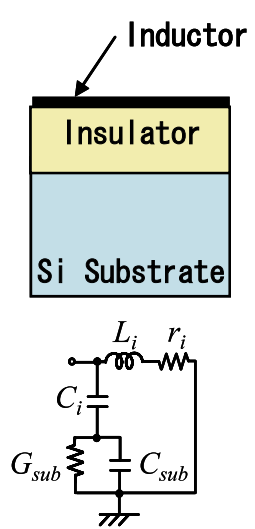

(a)

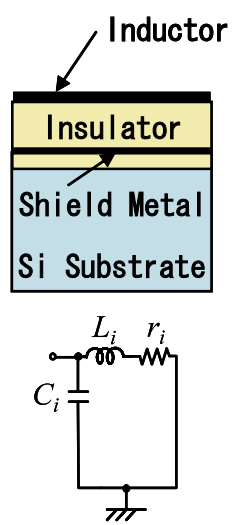

(b)

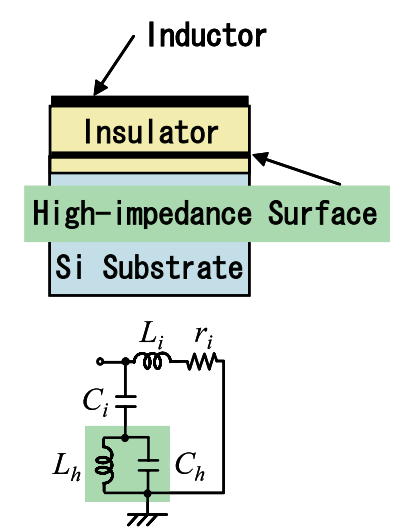

(c)

Fig. 1. Structures and equivalent circuits for inductors (a) without shield metal, (b) with shield metal, and (c) with a high-impedance surface. 
Figure 2 shows an implementation of the proposed inductor. The inductor $L_{i}$ is formed on layer $3(L 3)$. The HIS consists of the capacitance $C_{h}$, formed between $L 1$ and $L 2$, and the inductor $L_{h}$. The inductor $L_{h}$ may be formed on $L 2$, but in ordinary CMOS LSI's the lower layers are thinner than the upper layers and therefore have sheet resistances that are larger than those of the upper layers. The parasitic resistance $r_{h}$ is thus lower when $L_{h}$ is placed on L3 as shown in Fig. 2.

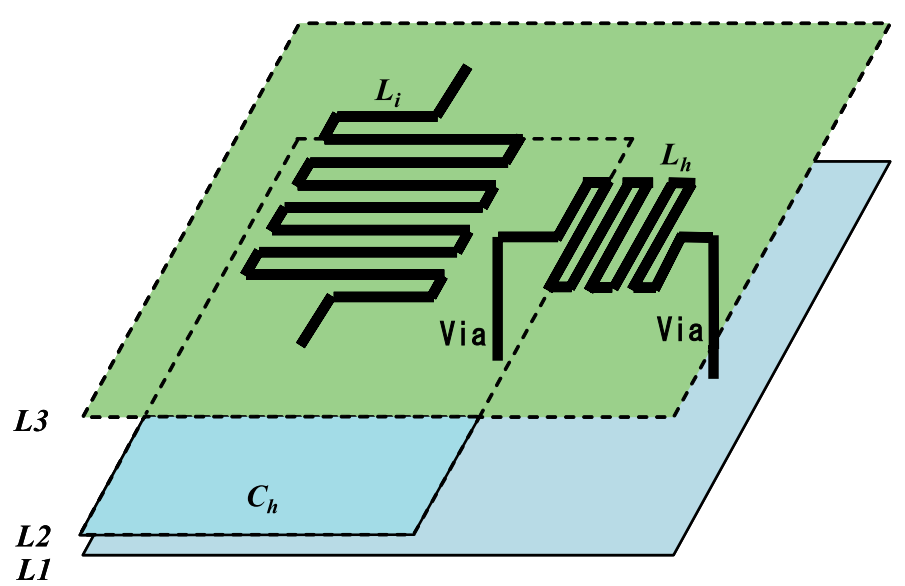

Fig. 2. Implementation of proposed inductor.

\section{Calculation results}

The characteristics of inductors with a HIS were compared with those of conventional inductors by using the equivalent circuits shown in Figs. 1 (b) and (c) and taking into account the parasitic resistance $r_{h}$. For simplicity, both inductors ( $L_{i}$ and $L_{h}$ in Fig. 2) were treated as straight lines and values of inductances and capacitances were obtained assuming that the metal thickness was zero. The sheet resistance of inductors was assumed to be $9 \mathrm{~m} \Omega / \square$, which is a feasible sheet resistance for $2-\mu$ m-thick $\mathrm{Cu}$ [1]. The insulator between $L 2$ and $L 3$ was assumed to have a relative dielectric constant of 3 and to be $3.5 \mu \mathrm{m}$ thick. The distance between the conventional inductor and shield metal was assumed to be $4.5 \mu \mathrm{m}$. Other values used in calculations were as follows: $L_{i}=0.42 \mathrm{nH}, C_{i}=60 \mathrm{fF}, r_{i}=2.6 \Omega$ (inductor with HIS) or $2 \Omega$ (conventional inductor), $L_{h}=0.21 \mathrm{nH}, C_{h}=0.16 \mathrm{pF}$, and $r_{h}=1.3 \Omega$.

The calculated $Q$ and effective inductances $L_{\text {eff }}$ are shown in Fig. 3. The conventional inductor had a $Q$ of 15.9 and an $L_{\text {eff }}$ of $0.64 \mathrm{nH}$ at $18.5 \mathrm{GHz}$. In the inductor with a HIS and no $r_{h}$, the resonance determined by $L_{h}\left(C_{i}+C_{h}\right)$ appeared at about $23 \mathrm{GHz}$, and the $Q$ exceeded 20 between $23 \mathrm{GHz}$ and $31 \mathrm{GHz}$. The inductor with a HIS and a $r_{h}$ of $1.3 \Omega$ had a $Q$ of 11.3 and an $L_{e f f}$ of $0.65 \mathrm{nH}$ at $31 \mathrm{GHz}$. The resonance frequency determined by $L_{i} C_{i}$ was increased from $31.6 \mathrm{GHz}$ for the conventional inductor to $41 \mathrm{GHz}$. The reason for this is being investigated.

Although the peak $Q$ for the propose inductor is smaller than that for the conventional inductor, the high-impedance surface in the proposed inductor 
enable the operating frequency to be increased. The performance of the proposed inductor may be improved by optimizing circuit parameters.

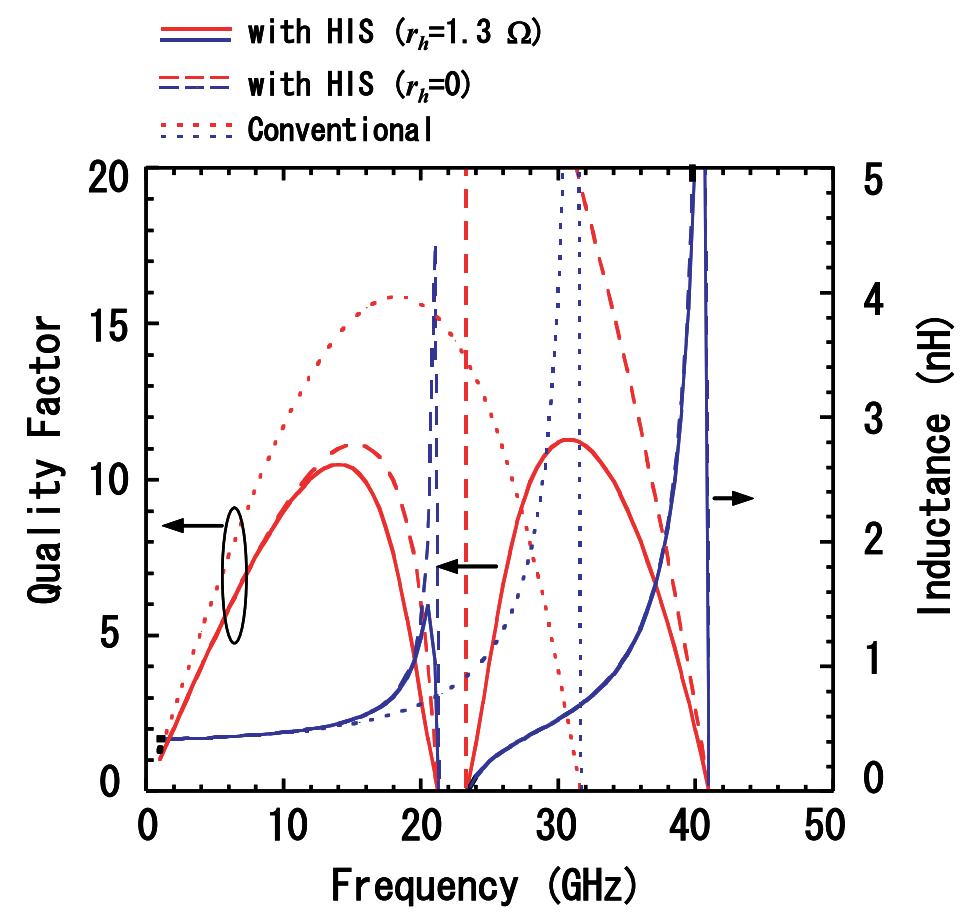

Fig. 3. Calculated quality factors and effective inductances.

\section{Conclusion}

A high-performance inductor for RF CMOS proposed here is based on the concept of a high-impedance surface. The inductor is shielded from the silicon substrate by a high-impedance surface consisting of a lumped capacitor and inductor. Theoretical calculations comparing the proposed inductor with a conventional inductor shielded by an ordinary metal surface show that the proposed inductor has a higher resonant frequency and quality factor $(Q)$ in the millimeter-wave region. The concept of the high-impedance surface can also be applied to high-impedance transmission lines on CMOS LSI's.

\section{Acknowledgments}

This work was partly supported by a Grant-in-Aid for Scientific Research from the Japanese Ministry of Education, Culture, Sports, Science and Technology. 